\title{
Establishing the HLS-Q12 short version of the European Health Literacy Survey Questionnaire: latent trait analyses applying Rasch modelling and confirmatory factor analysis
}

Hanne Søberg Finbråten ${ }^{1,2^{*}}$ (D), Bodil Wilde-Larsson ${ }^{2,3}$, Gun Nordström³ ${ }^{3}$ Kjell Sverre Pettersen ${ }^{4}$, Anne Trollvik ${ }^{3}$ and Øystein Guttersrud ${ }^{5}$

\begin{abstract}
Background: The European Health Literacy Survey Questionnaire (HLS-EU-Q47) is widely used in assessing health literacy $(\mathrm{HL})$. There has been some controversy whether the comprehensive HLS-EU-Q47 data, reflecting a conceptual model of four cognitive domains across three health domains (i.e. 12 subscales), fit unidimensional Rasch models. Still, the HLS-EU-Q47 raw score is commonly interpreted as a sufficient statistic. Combining Rasch modelling and confirmatory factor analysis, we reduced the 47 item scale to a parsimonious 12 item scale that meets the assumptions and requirements of objective measurement while offering a clinically feasible HL screening tool. This paper aims at (1) evaluating the psychometric properties of the HLS-EU-Q47 and associated short versions in a large Norwegian sample, and (2) establishing a short version (HLS-Q12) with sufficient psychometric properties.

Methods: Using computer-assisted telephone interviews during November 2014, data were collected from 900 randomly sampled individuals aged 16 and over. The data were analysed using the partial credit parameterization of the unidimensional polytomous Rasch model (PRM) and the 'between-item' multidimensional PRM, and by using one-factorial and multi-factorial confirmatory factor analysis (CFA) with categorical variables.

Results: Using likelihood-ratio tests to compare data-model fit for nested models, we found that the observed HLSEU-Q47 data were more likely under a 12-dimensional Rasch model than under a three- or a one-dimensional Rasch model. Several of the 12 theoretically defined subscales suffered from low reliability owing to few items. Excluding poorly discriminating items, items displaying differential item functioning and redundant items violating the assumption of local independency, a parsimonious 12-item HLS-Q12 scale is suggested. The HLS-Q12 displayed acceptable fit to the unidimensional Rasch model and achieved acceptable goodness-of-fit indexes using CFA.

(Continued on next page)
\end{abstract}

\footnotetext{
* Correspondence: hanne.finbraten@inn.no

${ }^{1}$ Department of Public Health and Department of Nursing, Faculty of Social and Health Sciences, Inland Norway University of Applied Sciences, PO Box 400, N-2418 Elverum, Norway

${ }^{2}$ Department of Health Sciences, Faculty of Health, Science and Technology,

Nursing science, Karlstad University, SE-65188 Karlstad, Sweden

Full list of author information is available at the end of the article
}

(c) The Author(s). 2018 Open Access This article is distributed under the terms of the Creative Commons Attribution 4.0 International License (http://creativecommons.org/licenses/by/4.0/), which permits unrestricted use, distribution, and reproduction in any medium, provided you give appropriate credit to the original author(s) and the source, provide a link to the Creative Commons license, and indicate if changes were made. The Creative Commons Public Domain Dedication waiver (http://creativecommons.org/publicdomain/zero/1.0/) applies to the data made available in this article, unless otherwise stated. 
(Continued from previous page)

Conclusions: Unlike the HLS-EU-Q47 data, the parsimonious 12-item version (HLS-Q12) meets the assumptions and the requirements of objective measurement while offering clinically feasible screening without applying advanced psychometric methods on site. To avoid invalid measures of HL using the HLS-EU-Q47, we suggest using the HLSQ12. Valid measures are particularly important in studies aiming to explain the variance in the latent trait $H L$, and explore the relation between $\mathrm{HL}$ and health outcomes with the purpose of informing policy makers.

Keywords: Confirmatory factor analysis of categorical data, Health literacy, HLS-EU-Q47, HLS-Q12, Rasch modelling, Short version, Validation

\section{Background}

Health literacy (HL) is believed to have a vital impact on public health, including access to and the use of health services [1-3]. Low HL is associated with poorer health conditions $[3,4]$, more frequent use of health services, longer hospitalisations [2, 3] and higher mortality $[4,5]$. Further, some studies relate low HL to unhealthy behaviours, such as smoking [1, 6-9], low physical activity [7-12] and less use of preventive services [1, 4, 7]. Several instruments have been developed to measure individuals' HL and explore how health outcomes relate to HL. Some instruments assess basic reading and writing skills (such as the Test of Functional HL in Adults [TOFHLA] [13] and the Rapid Estimate of Adult Literacy in Medicine [REALM] [14]), while others assess specific health and cognitive domains (such as the European Health Literacy Survey Questionnaire [HLS-EU-Q47] [11, 15]). The HLS-EU-Q47 was developed from a conceptual model covering four cognitive domains within three health domains, which constitutes a 12-cell matrix for item development [16]. The cognitive domains comprise the proficiency to access (A), understand (B), appraise (C) and apply (D) health information. These competencies are considered necessary to handle health information within health care $(\mathrm{HC})$, disease prevention (DP) and health promotion (HP) settings.

The psychometric properties of the HLS-EU-Q47 have been investigated using principal component analysis (PCA) [15] and confirmatory factor analysis (CFA) [17-19]. When applying CFA in these studies, the health domains were treated as three uncorrelated or orthogonal subscales confirming a four-component structure reflecting the four cognitive domains. A Norwegian study among individuals with type 2 diabetes [20], in which the HLS-EU-Q47 was validated using Rasch-modelling in combination with CFA, revealed that the three health domains were highly correlated and that a 12-dimensional model (reflecting the four cognitive domains across the three health domains in the conceptual framework) obtained the best data-model fit. These results were later confirmed in a Taiwanese study among stroke patients [21]. A 12-dimensional scale requires a multidimensional approach and provides 12 different scores for each individual, which is impractical in clinical settings. Despite the theoretically identified and empirically confirmed multidimensionality of the HLS-EU-Q47, several studies assume a unidimensional latent variable and report one overall HL sum score [3, 9, 11, 17-19, 22-24]. Beside multidimensionality, researchers have observed items with poor fit, redundant items, items displaying unordered response categories and subscales with low precision or reliability [20, 21]. Therefore, we aim to clarify these issues based on a large sample of randomly selected individuals.

A unidimensional short version of the HLS-EU-Q47 will provide clinically useful data in a fast, reliable and accurate manner without applying advanced psychometry. Moreover, measurement scales should be anchored in theory and conceptual frameworks [25]. Hence, it is desirable that the short version reflects the conceptual HL model of Sørensen et al. [16], and that the 12 domains are equally represented. In addition, nurses and other health professionals should be able to use the short version to map patients' HL without applying advanced psychometric approaches. Several short versions of the HLS-EU-Q47 have been suggested, such as the HLS-EU-Q16 [11] and HL-SF12 [26]. However, we did not find any peer-reviewed publications describing the basis on which the items were selected, whether these short versions were validated using Rasch modelling, or whether they are sufficiently unidimensional. Therefore, based on the findings of Finbråten et al. [20] and the validation procedure in the present study, Rasch modelling and CFA are used to establish a unidimensional 12 item version of the HLS-EU-Q47.

Rasch models meet the assumptions and requirements of fundamental measurement, such as additivity [27], specific objectivity [28], sufficiency [29] and invariance [30]. Both Rasch modelling and CFA can be considered appropriate methods for assessing dimensionality [31, 32]. CFA is used at an overall level to assess discriminant validity (dimensionality) and factorial validity (loadings or common variance), whereas Rasch modelling in addition provide detailed information at the item level, in addition to assessing local independency.

Against this background, the aims of our paper are to: (1) evaluate the psychometric properties of the HLS-EU-Q47 and associated short versions in the Norwegian population 
and (2) establish a parsimonious unidimensional short version (HLS-Q12) with sufficient psychometric properties. More specifically, we will test the following falsifiable hypothesis: When applied to Norwegian adults, the HLS-EU-Q47 represents a unidimensional, well-targeted scale with acceptable person separation (reliability), consisting of independent and invariant items at the ordinal level (i.e., ordered response categories) each displaying sufficient fit to the partial-credit parameterisation of the unidimensional polytomous Rasch-model.

The same hypothesis is tested for the HLS-Q12, and is used as a basis for comparing the psychometric properties of HLS-Q12 to the HL-SF12 and the HLS-EU-Q16.

\section{Methods}

\section{Data collection}

A random sample of 900 Norwegians aged 16 and over responded to the HLS-EU-Q47 during November 2014. For comparative purposes, we initially aimed at applying identical data collection procedures as the European HL survey [11], but owing to personal resources, it was not possible to perform face-to-face interviews. Hence, data were collected using computer-assisted telephone interviews. The international survey agency, Ipsos, with access to country representative samples, collected the data following detailed instructions offered by the researchers. The European HL survey sampled approximately 1000 respondents in each participating country [11] independent of population size. As there are significantly fewer inhabitants in Norway compared to most of the countries that participated in the European HL survey [33], 900 were sampled. The age limit was set to 16 years as this is the age of licence in relation to health in Norway.

\section{Measures}

The measures included the 47 items HLS-EU-Q47 HL survey questionnaire and additional person factors, such as gender, age and highest level of education completed.

\section{The HLS-EU-Q47}

Sørensen et al. [11, 15] developed the HLS-EU-Q47 items to reflect their conceptual model, and suggested a 4-point rating scale with response categories ranging from very easy (1) to very difficult (4). In the present study, the rating scale was reversed to make a higher score indicate a higher proficiency at the latent trait. A 'don't know' category, which was later recoded to missing data, was added to record such spontaneous utterings from respondents during the telephone interviews.

\section{Translation of the HLS-EU-Q47}

The translation procedure, that involved forward and back translation, is thoroughly described in Finbråten et al. [20]. Cognitive interviews were used to explore item interpretation and clarify any ambiguities.

\section{Data analysis}

Rasch modelling was used to assess the psychometric properties of the HLS-EU-Q47 and as a basis for developing the HLS-Q12. Rasch modelling and CFA were used to study the psychometric properties of the short versions HLS-Q12 (our suggested short version), HL-SF12 [26] and the HLS-EU-Q16 [11].

The data were analysed up against the partial-credit parameterisation [34] of the unidimensional polytomous Rasch model (PRM) and the 'between-item' multidimensional PRM $[35,36]$. Below, the one-dimensional approach refers to unidimensional Rasch analysis. The two- and three-dimensional approaches refer to an oblique or unrestricted multidimensional Rasch analysis where the health domains are allowed to covary. The two-dimensional approach corresponds to the HC subscale and the combined DP and HP subscales, whereas the three-dimensional approach reflects the three health domains. The 12-dimensional approach refers to a similar analysis, where the four cognitive domains and the three health domains define 12 correlated subscales.

The RUMM2030 statistical software package [37] was used for the one-dimensional and consecutive Rasch analyses (treating the subscales as orthogonal or uncorrelated), and the ConQuest 4 statistical software package [38] was used for the one-dimensional and multidimensional Rasch analyses. CFA was performed using LISREL9.3 software [39].

\section{Rasch modelling}

The null hypothesis of a locally independent scale is weakened and might be rejected in favour of the alternative hypothesis, suggesting a multidimensional scale, in the following situations: When the proportion of individuals with significantly different person-location estimates on a pair of related subscales exceeds $5 \%$ (or the lower bound of the binominal 95\% confidence interval (CI) exceeds 5\%) [40-43]; when the subtest analysis in RUMM points to low common subscale variance and high unique subscale variance (i.e. low fractal index $A<0.8$ and high fractal index $c$ close to 0.5 , respectively) [44]; when a multidimensional Rasch model obtains a better data-model fit than a one-dimensional Rasch model (i.e. significantly lower deviance or -2log-likelihoods using a likelihood-ratio test for nested models and AIC for non-nested models) [45]; and/or when absolute values of Rasch-model residual correlations among items exceed 0.3 [46].

Likelihood-ratio tests (LRT) were used to compare data-model fit for nested models, such as the one-, two-, three- and 12-dimensional Rasch models, where the first 
implies parallel subscales (correlations between all theoretically defined subscales were fixed at 1) and the latter implies freely correlated subscales. The LRT statistic "difference in deviance" is asymptotically $x 2$ distributed with degrees of freedom $(d f)$ equal to the difference in the number of estimated model parameters comparing two nested models [47]. Akaike's information criterion (AIC) [48] was used to compare the data-model fit for non-nested models, such as the various short versions. Similar to Allen and Wilson [49], both deviance and AIC are reported when applying the consecutive approach to the HLS-EU-Q47 (calculated by adding the values for each of the three subscales). Lower values of deviance and AIC indicate better data-model fit. In addition to deviance and AIC, total item chi-square with $p$-values was used to assess the data-model fit of the short versions.

Items were interpreted as under-/over-discriminating relative to the Rasch model when the infit MNSQ were significantly different from the expected value of 1 (indicating perfect fit to model) or, more precisely, when the infit value was above/below the $95 \% \mathrm{CI}$ and the corresponding $t$ value was $>1.96[45,50]$.

In addition to infit, chi-square probability was used to assess item fit for the various short versions. Items were considered as misfitting at high chi-square values and probability values lower than a Bonferroni-adjusted 5\% [51]. Item difficulty was described using item-location estimates.

Applying the consecutive approach to the HLS-EU-Q47 and the one-dimensional approach to the short versions, differential item functioning (DIF) was explored using analysis of variance (ANOVA) [40] for the dichotomized person factors of gender, age and level of highest completed education: age was dichotomised as above or below the sample mean (47 years), and highest level of completed education was split into "compulsory and upper secondary school" versus "university level".

The ordering of the response categories was examined applying the one-dimensional and the consecutive approaches. Significantly different thresholds in "correct" order [52, 53] were used as evidence for ordered response categories. The targeting of the (sub)scales was evaluated by comparing the distribution of the item threshold estimates to the distribution of the person estimates [51]. For more detailed descriptions of the analyses performed, see for example, Andrich [30], Hagquist [53], Hagquist, Bruce and Gustavsson [54] or Finbråten et al. [20].

\section{Confirmatory factor analysis}

The null hypothesis of a one-dimensional scale is weakened when overall fit indexes for multifactorial CFA are superior to a one-factorial CFA [55]. Owing to categorical raw data, we defined the variables as ordinal in the "lsf" file and estimated the polychoric item correlations and their asymptotic covariance matrix using the PRELIS application to LISREL [56]. As target values for goodness-of-fit (GOF) indexes is based on maximum likelihood estimation (ML), we used robust ML to obtain the Satorra-Bentler scaled chi-square GOF index $\left(\mathrm{SB}\right.$ scaled $\left.X^{2}\right)$ [57, 58]. The following overall GOF indexes for model comparison based on the null model were used: SB scaled $\chi^{2}$, standardized root mean square residual (SRMR), root mean square error of approximation (RMSEA), comparative fit index (CFI) and non-normed fit index (NNFI, or Tucker-Lewis index [TLI]). Schumacker and Lomax [59] recommend SRMR $<.05$, RMSEA $\leq .05$, and CFI and NNFI $\geq .95$ as GOF index target values, whereas $\mathrm{Hu}$ and Bentler [60] claim that SRMR and RMSEA values close to or below 0.8 and 0.6, respectively, indicate sufficient overall fit. The $\mathrm{AIC}$ and the Bayesian information criterion (BIC) were also taken into account. AIC and GOF indexes were used to compare the various short versions and to compare the one-factor to the three-factor approaches for the various short versions. Item communalities (squared factor loadings for completely standardized solution) describe the shared variance among the items that is accounted for by the latent variable, HL [61].

\section{Reliability}

Person separation index (PSI), person separation reliability (PSR) and the $H$ coefficient [62] (obtained from RUMM, ConQuest and calculated manually based on communalities from CFA) were used as measures of reliability. Values exceeding $0.85(0.65)$ are sufficient if conclusions are to be drawn at the individual (group) level [63].

\section{Developing a one-dimensional short version}

The suggested short version in the present study, the HLS-Q12, was developed from analyses of the HLS-EU-Q47 in two Norwegian samples; people with type 2 diabetes [20] and the general population. The suggested version, HLS-Q12, was developed by stepwise exclusion of poorly fitting items, items displaying DIF and items collecting redundant information, together with a qualitative evaluation of the item content in light of the conceptual framework. For dependent items, the item with the most essential information was retained, while the other item was discarded. We ensured that the items in our final scale (specified in Table 5) were distributed across the HLS-EU 12-cell matrix $[11,16]$.

\section{Missing data}

The dataset we analysed was complete, but there was, on average, 29 (3\%) 'don't know' responses per item, which 
we recoded as missing values. Item 2 had the highest number of 'don't know' responses (69 or 8\%). Rasch modelling was performed on incomplete data (except in the subtest analyses), whereas LISREL used list-wise deletion as the default method, reducing the sample size from 900 to effective sample sizes of 699,680 and 670 for the HLS-Q12, HL-SF12 and the HLS-EU-Q16, respectively.

\section{Results}

The sample $(n=900)$ distributions for gender and age reflected the population distributions, while individuals with higher education were somewhat over-sampled (Table 1).

\section{Psychometric properties of the HLS-EU-Q47 Overall analyses of HLS-EU-Q47}

Overall analyses included those for dimensionality, data-model fit, response dependence and reliability. The initial analyses of dimensionality indicated that neither

Table 1 Characteristics of study sample and the Norwegian population

\begin{tabular}{lll}
\hline & $\begin{array}{l}\text { Sample } \\
(n=900)\end{array}$ & $\begin{array}{l}\text { Population } \\
(\mathrm{N}=4,115,195)\end{array}$ \\
\hline Age & $47.0 \pm 17.7$ & $46.5 \pm 19.0$ \\
mean \pm sd & 48 & 45 \\
- median & $16-91$ & $16-105$ \\
- min-max & 0 & 0 \\
- missing & $\%$ & $\%$ \\
& 13 & 15 \\
16-24 years & 25 & 25 \\
25-39 years & 25 & 26 \\
40-54 years & 21 & 17 \\
55-66 years & 14 & 12 \\
67-79 years & 2 & 5 \\
80+ years & $n(\%)$ & $n(\%)$ \\
Gender & $441(49)$ & $2,567,434(50)$ \\
- males & $459(51)$ & $2,541,622(50)$ \\
- females & 0 & \\
- missing & $n(\%)$ & $\%$ \\
Highest completed education & $87(10)$ & 26 \\
- compulsory comprehensive school & $298(33)$ & 38 \\
- upper-secondary school & $321(36)$ & 23 \\
- university level, lower degree ${ }^{\text {b }}$ & $191(21)$ & 9 \\
- university level, higher degree & & \\
- missing & $3(<1)$ & \\
\hline
\end{tabular}

${ }^{a}$ Norwegian population 16 years and over in 2014 (Statistics Norway, 2017) ${ }^{\mathrm{b}}$ Education at university or university college for 4 years or less (Bachelor's degree)

'Education at university or university college for 5 years or more (Master's degree and/or PhD) the entire HLS-EU-Q47 nor the three health domains, HC, DP and HP, were sufficiently unidimensional (the respective proportions of significant $t$-tests were 21,9 , 13 and 10\% [not reported in Table 2]).

Using LRT, the observed data were more likely under a 12-dimensional Rasch model than a one-, two- or three-dimensional Rasch model (Fig. 1). As expected, the data-model fit improved significantly in each step from the one-dimensional through the 12-dimensional model. For example, the drop in deviance from the three- to the 12-dimensional model was $\Delta D=3878 \approx \chi^{2}[\Delta d f=\Delta$ estimated parameters $(\mathrm{ep})=72], p<0.01$, critical value $=103$. The three-dimensional model had a lower AIC and a better fit than the consecutive approach of the three health domains, HC, DP and HP $(\triangle \mathrm{AIC}=1129)$. The correlations between $\mathrm{HC}$ and DP, HC and HP, and DP and HP were $r=0.81,0.71$ and 0.83 , respectively.

Applying the one-dimensional approach, we observed nine pairs of dependent items ( 1 and 2, 22 and 23, 41 and 42, 44 and 46, 45 and 47, 3 and 7, 12 and 28, 17 and 21,30 and 37 ), of which the latter four pairs surprisingly appeared across the theoretically defined subscales. Applying the consecutive approach to the three health domains, only one pair of dependent items was observed within each health domain (1 and 2, 17 and 21, and 41 and 42 , respectively).

Applying the 12-dimensional approach to the HLS-EU-Q47, we observed relatively low subscale reliability indexes, most likely owing to few items per scale-on average 3.9 items per subscale (see PSR values in Fig. 1). Consequently, we observed acceptable reliability indexes for the one-dimensional approach and for the subscales of the two- and the three-dimensional approaches.

\section{Analyses of HLS-EU-Q47 at item level}

Analyses at the item level included analyses of item fit, item discrimination, DIF and ordering of response categories. Table 2 shows the item fit when applying the one-, three- and 12-dimensional approaches.

Under-discriminating items were observed using the one- (items 12, 29, 38, 45 and 47), three- (items 11, 12, 29, 30 and 38) and 12-dimensional (items 12, 18, 19, 38 and 43) approaches; items 12 and 38 under-discriminated in all analysis approaches. Over-discriminating items were also observed when the one- (items 16, 19, 20 and 40), three- (items 8 and 40) and 12- dimensional (item 16) approaches were applied. Using the consecutive approach, several items displayed DIF (Table 2).

Unordered response categories were observed for items 8,15 and 21 when applying the one-dimensional approach, as well as when analysing the $\mathrm{HC}$, DP and $\mathrm{HC}$ health domains consecutively (Table 2). 


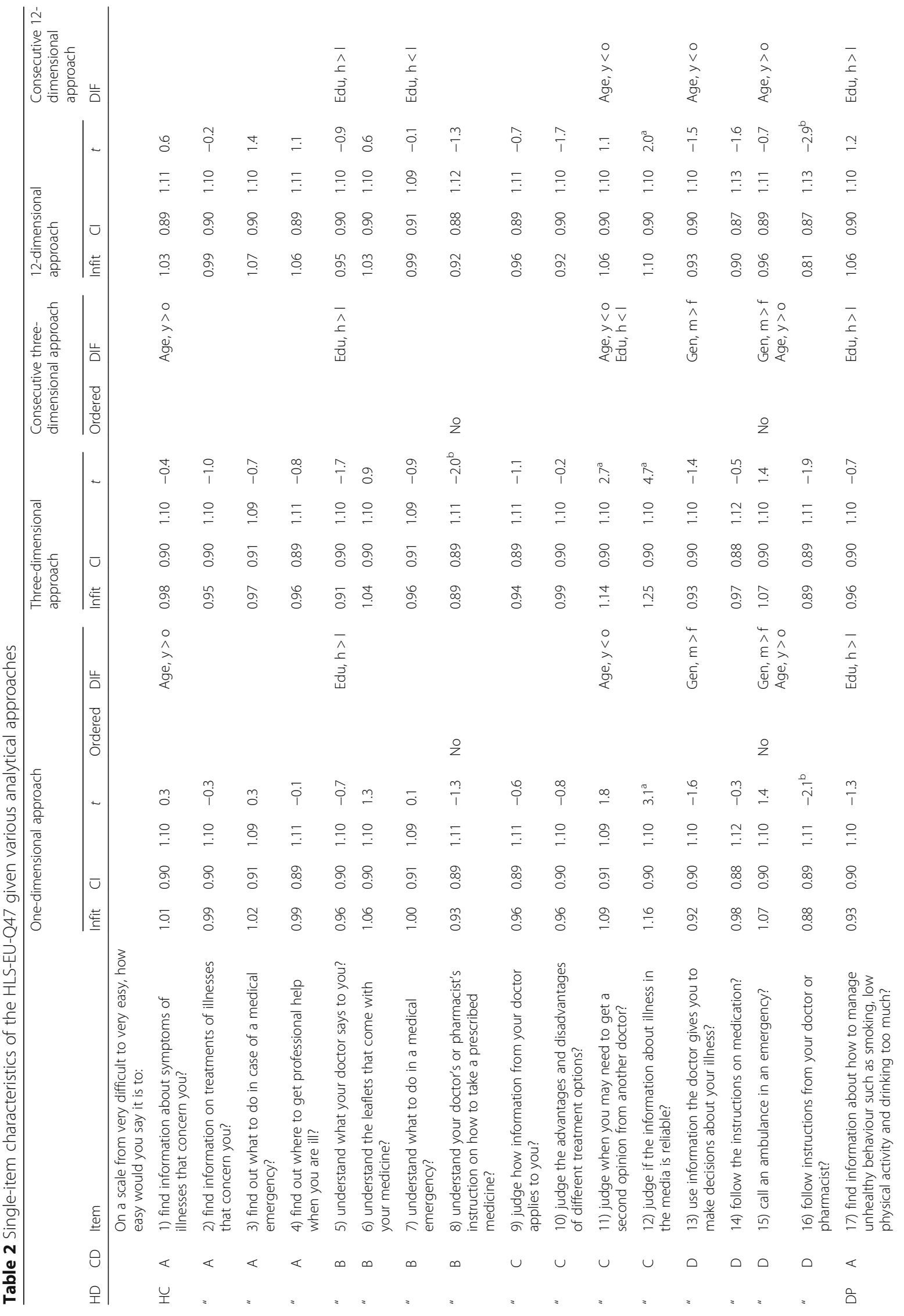




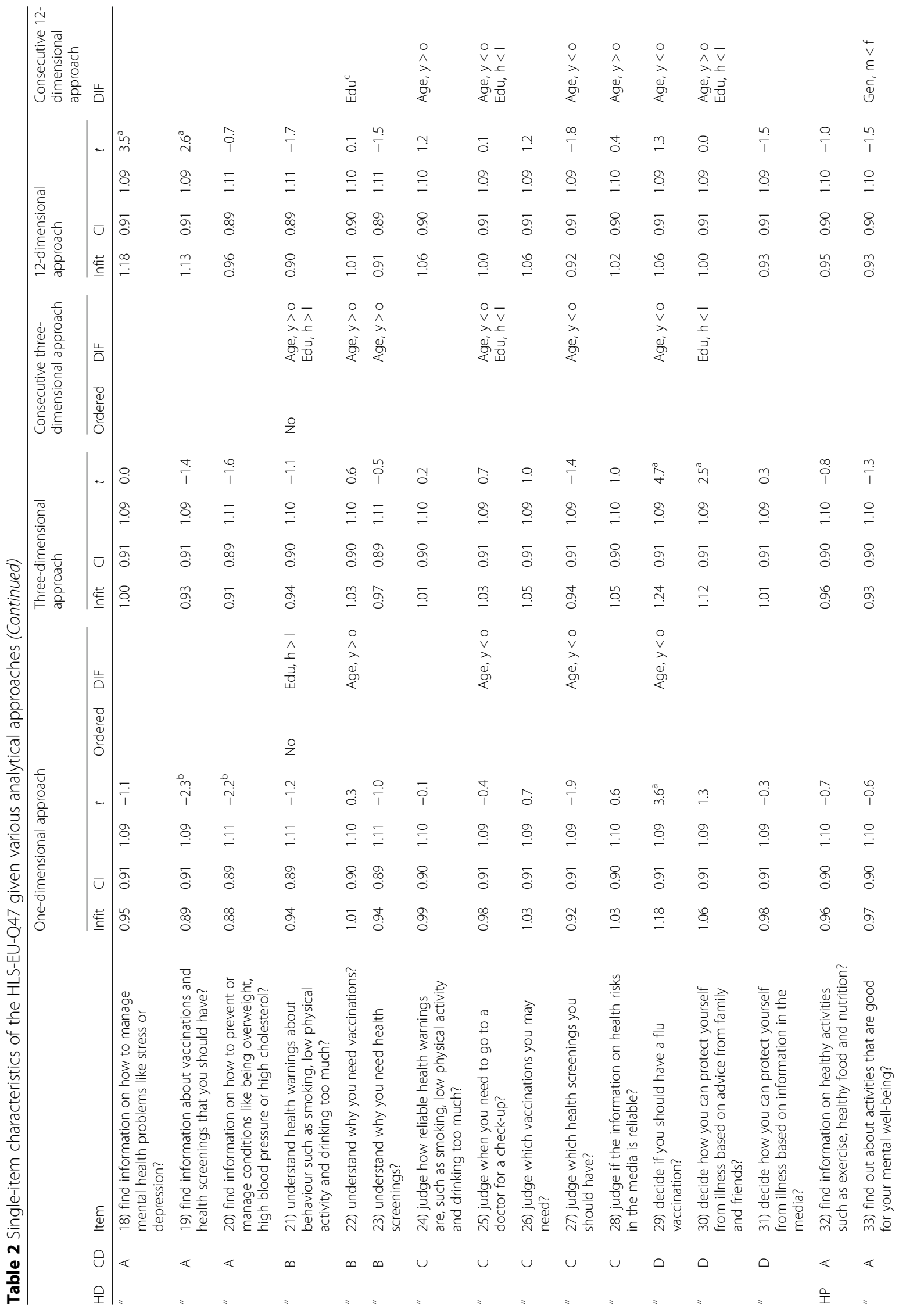




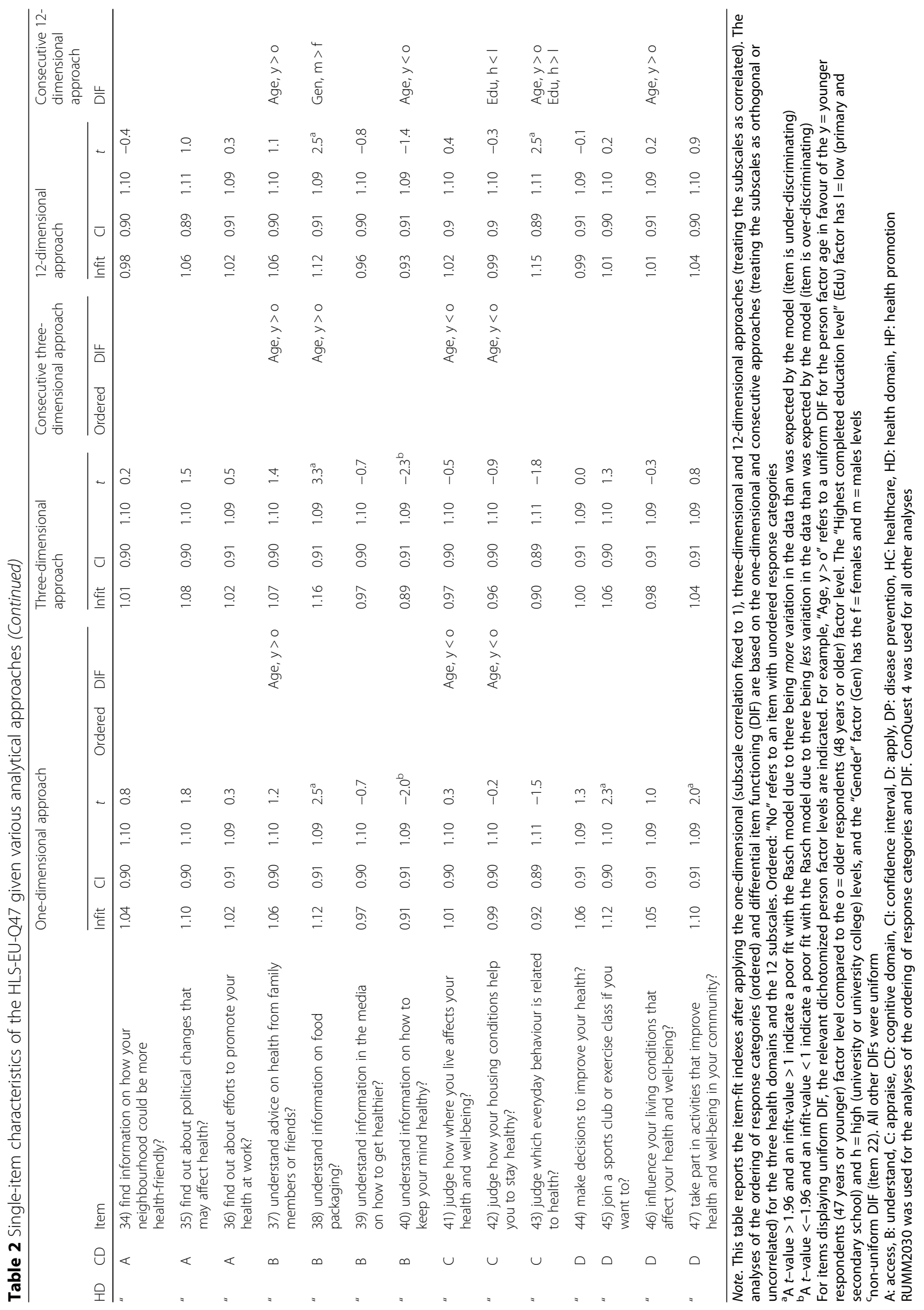




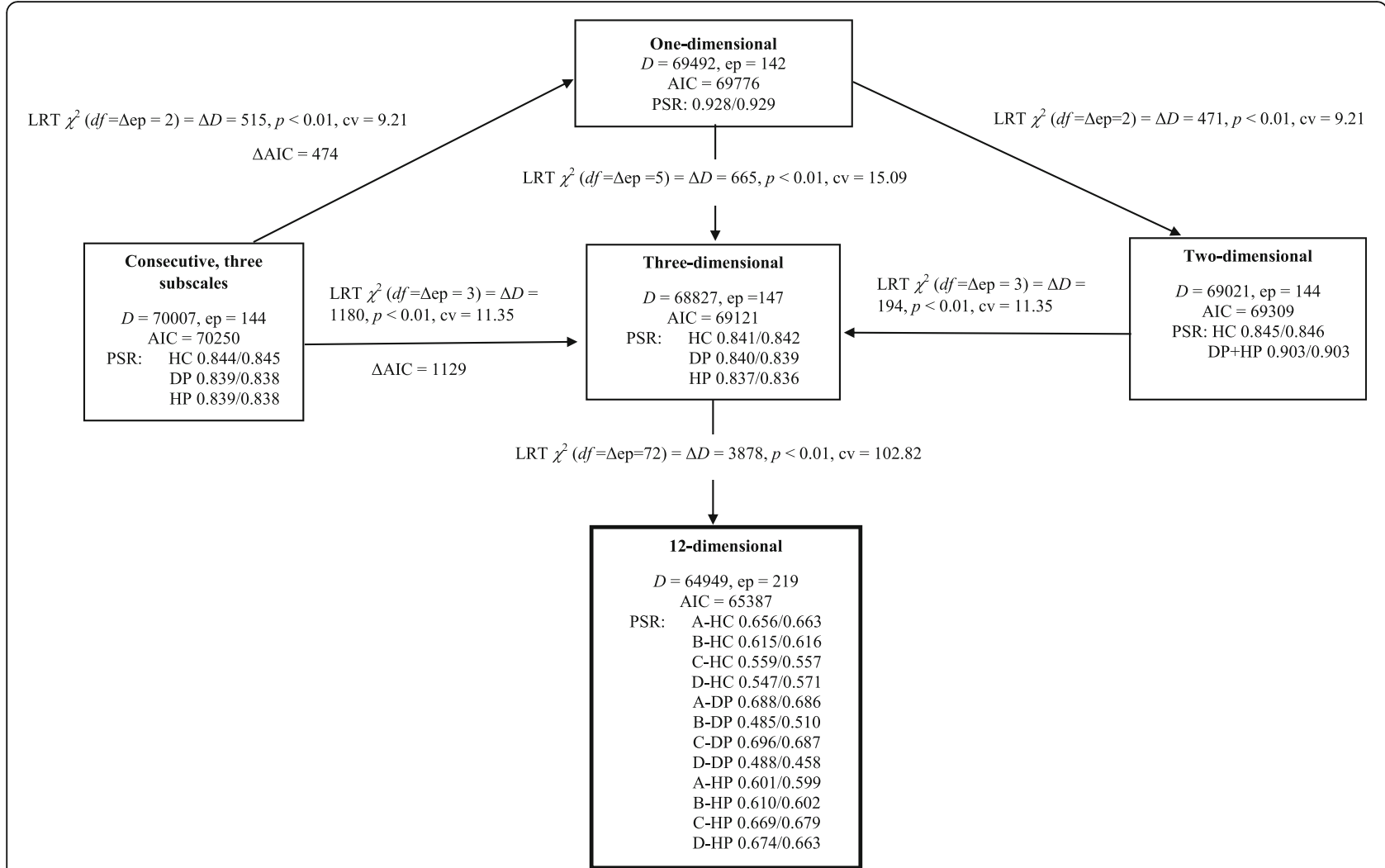

Fig. 1 Model fit of the HLS-EU-Q47 after applying various analysis approaches. Figure 1 shows the overall fit statistics for the one-dimensional approach (all subscale correlations fixed to 1), the consecutive approach (treating the three health domains as orthogonal or uncorrelated) and the two-, three- and 12-dimensional approaches (treating the theoretical subscales as correlated). A: access, B: understand, C: appraise, D: apply (cognitive domains). HC: health care, DP: disease prevention, HP: health promotion (health domains). $\Delta$ : change in parameter, AIC: Akaike's information criterion, cv: critical value, D: deviance, $d f$ : degrees of freedom, ep: number of estimated parameters, LRT: likelihood ratio test. PSR: person separation reliability based on marginal maximum likelihood estimate/Warm's mean weighted likelihood estimate

Psychometric properties of the HLS-EU-Q47 short versions HLS-Q12, HL-SF12 and HLS-EU-Q16

Overall analyses of the HLS-Q12, HL-SF12 and HLS-EU-Q16

According to the $t$-tests, none of the short versions could be deemed sufficiently unidimensional (the proportions of significant $t$-tests were above $5 \%$ and the lower binominal 95\% CI proportion was 0.07 for both the HLS-Q12 and the HL-SF12, whereas the lower binominal 95\% CI-proportion was 0.09 for the HLS-EU-Q16 (Table 3).

Nevertheless, the fractal indexes indicated that the HLS-Q12 and HL-SF12 had relatively high amounts of common variance $(A=0.90$ and 0.91 , respectively), high subscale correlation ( $r=0.80$ and 0.87 , respectively), and relatively high unique subscale variance $(c=0.50$ and 0.39 , respectively).

Using LRT, the data fit better to the three-dimensional models compared to the one-dimensional models of the various short versions (three-dimensional models had significantly lower deviance, except for the HL-SF12, where the difference was insignificant). The GOF indexes obtained via CFA were also better for the three-factor models as compared to the one-factor models of the various short versions (Table 4).

Comparing the short versions, the HLS-Q12 obtained best fit to the Rasch model (had the lowest total item chi-square value and the lowest AIC; Table 3) whereas the HL-SF12 obtained best fit when performing CFA (had the lowest likelihood estimates and the best GOF indexes). The HLS-Q12 also had acceptable GOF indexes (Table 4). The HLS-EU-Q16 displayed the weakest overall fit indexes (highest total item $\chi^{2}$ value and highest log likelihood estimates).

All the one-dimensional short versions had acceptable reliability values (PSI and PSR values above 0.75 and the $H$ coefficient was above 0.82 ). The HLS-EU-Q16 had the highest reliability indexes $(\mathrm{PSI}=0.830, \mathrm{PSR}=0.826$ and $\mathrm{H}=0.882$ ). The reliability indexes were slightly higher for the HLS-Q12 than for the HL-SF12 (Tables 3 and 4). Applying the three-dimensional approach, the PSR and $H$ values of the correlated subscales were below the recommended values (Tables 3 and 4). The HLS-Q12 was the best-targeted, with a mean person location of 0.759. The mean person locations for the HL-SF12 and 
Table 3 Unidimensionality, data-model fit and reliability applying Rasch modelling of the various short versions

\begin{tabular}{|c|c|c|c|c|c|c|}
\hline & \multicolumn{2}{|l|}{ HLS-Q12a } & \multicolumn{2}{|l|}{ HL-SF12 [26] } & \multicolumn{2}{|l|}{ HLS-EU-Q16 [11] } \\
\hline & One-dimensional & $\begin{array}{l}\text { Three- } \\
\text { dimensional }\end{array}$ & One-dimensional & $\begin{array}{l}\text { Three- } \\
\text { dimensional }\end{array}$ & One-dimensional & $\begin{array}{l}\text { Three- } \\
\text { dimensional }\end{array}$ \\
\hline \multicolumn{7}{|l|}{ Unidimensionality } \\
\hline $\begin{array}{l}\text { Proportion (\%) of significant } \\
t \text {-tests (lower Cl-prop) }\end{array}$ & $8.78 \%(0.07)$ & & $8.01 \%(0.07)$ & & $10.44 \%$ (0.09) & \\
\hline PSI & 0.767 & & 0.759 & & 0.830 & \\
\hline$P S I^{b}$ & 0.687 & & 0.668 & & 0.703 & \\
\hline Fractal indexes & & na & & na & & na \\
\hline c & 0.50 & & 0.39 & & 0.46 & \\
\hline$A$ & 0.90 & & 0.91 & & 0.85 & \\
\hline$r$ & 0.80 & & 0.87 & & 0.82 & \\
\hline $\begin{array}{l}\text { Total item chi square }(d f) \text {, } \\
\text { probability }\end{array}$ & $\begin{array}{l}112.61(108) \\
0.361\end{array}$ & & $\begin{array}{l}130.21(108), \\
0.072\end{array}$ & & $\begin{array}{l}208.90(144) \\
0.00034\end{array}$ & \\
\hline \multicolumn{7}{|l|}{ Log-likelihoods } \\
\hline Deviance (ep) & $18,590(37)$ & $18,556(42)$ & $18,707(37)$ & $18,696(42)$ & $22,964(49)$ & $22,815(54)$ \\
\hline AIC (ep) & $18,664(37)$ & $18,640(42)$ & $18,781(37)$ & $18,780(42)$ & $23,062(49)$ & $22,923(54)$ \\
\hline \multicolumn{7}{|l|}{ Reliability } \\
\hline PSR (MLE) & 0.762 & $0.537 / 0.517 / 0.575$ & 0.758 & $0.501 / 0.497 / 0.564$ & 0.825 & $0.713 / 0.619 / 0.545$ \\
\hline \multicolumn{7}{|c|}{$\begin{array}{l}\text { Note. The table shows the results of the tests of unidimensionality based on paired } t \text {-tests of person-location estimates for subsets of items. It also reports fractal } \\
\text { indexes, } c, A \text { and } r \text {. The person separation index (PSI) and fractal indexes were estimated for the complete dataset (HLS-Q12 } n=696, \text { HL-SF12 } n=680, \text { HLS-EU-Q47 } \\
n=670) \text {. Significant } t \text {-tests } \leq 5 \% \text { (or lower confidence interval [CI] proportion } \leq 5 \% \text { ), small drops in PSI ( }{ }^{b} \text { after adjusting for violations of local independence due to } \\
\text { subtest structure), high values of } A \text { and } r \text {, and low values of } c \text { could indicate unidimensionality. Analyses were performed by using RUMM2030 software } \\
\text { Log-likelihoods and person separation reliability (PSR) were estimated for the one- and three-dimensional approaches to the short versions by using ConQuest } 4 \\
\text { software. Lower values of deviance and AIC indicate a better fit } \\
\text { AIC: Akaike's information criterion, ep: number of estimated parameters, na: not applicable, PSR (MLE): person separation reliability based on a marginal maximum } \\
\text { likelihood estimate } \\
\text { All the short versions are developed on the basis of the HLS-EU-Q47 } \\
\text { aHLS-Q12 developed through the present study }\end{array}$} \\
\hline
\end{tabular}

HLS-EU-Q16 were 0.816 and 1.020, respectively (not shown in the table).

\section{Analyses of HLS-Q12, HL-SF12 and HLS-EU-Q16 at the item level}

Compared with the HL-SF12 and the HLS-EU-Q16, the HLS-Q12 showed best fit at the item level as there were no occurrences of misfitting items, items displaying DIF with regard to available person factors or items with unordered response categories. For the HL-SF12, items were within-item biased (items 15, 26 and 30 displayed DIF), and one item with unordered response categories was observed (item 15). Problems with regard to DIF (items 5, 11, 13, 21 and 37) and unordered response categories (items 8 and 21) were also observed for the HLS-EU-Q16. In addition, item 11 under-discriminated and showed significant misfit (chi-square probability $=0.001$; Table 5). A high level of unique variance was observed for the items of the various short versions (relatively low communality values).

Investigating the item-location estimates of the HLS-Q12, items 28 (judge if the information on health risks in the media is reliable) and 10 (judge the advantages and disadvantages of different treatment options) had the highest location estimates (1.068 and 0.892, respectively) and were thus the hardest to endorse. Items 23 (understand why you need health screenings) and 32 (find information on healthy activities such as exercise, healthy food and nutrition) were the easiest to endorse (item-location estimates of -1.158 and -1.135 , respectively).

\section{Discussion}

Based on our national random sample $(n=900)$, we found that empirical evidence did not support our null hypothesis associated with the psychometric properties of the HLS-EU-Q47. By excluding poorly fitting items, items displaying DIF and items violating local independence, we succeeded in establishing a psychometrically sound parsimonious 12-item version (HLS-Q12). From a measurement point of view, we found that the HLS-Q12 outperformed other available short versions of the HLS-EU-Q47, such as the HL-SF12 and the HLS-EU-Q16.

\section{Psychometric properties of the HLS-EU-Q47 Overall analyses of HLS-EU-Q47}

A 12-dimensional model described the HLS-EU-Q47 data best. This result is perfectly in line with prior research [20, 21]. Hence, applying the HLS-EU-Q47 
Table 4 Overall fit and reliability using confirmatory factor analyses of the various short versions

\begin{tabular}{|c|c|c|c|c|c|c|}
\hline \multirow[b]{2}{*}{ GOF index (LISREL) } & \multicolumn{2}{|l|}{ HLS-Q12a } & \multicolumn{2}{|l|}{ HL-SF12 [26] } & \multicolumn{2}{|l|}{ HLS-EU-Q16 [11] } \\
\hline & One-dimensional & Three-dimensional & One-dimensional & Three-dimensional & One-dimensional & Three-dimensiona \\
\hline $\begin{array}{l}\text { SB scaled } x^{2}(d f) \\
x^{2} p \text {-value }\end{array}$ & $\begin{array}{l}142.71(54) \\
p<0.001\end{array}$ & $\begin{array}{l}112.96(51) \\
p<0.001\end{array}$ & $\begin{array}{l}93.73(54) \\
p<0.001\end{array}$ & $\begin{array}{l}83.71(51) \\
p<0.01\end{array}$ & $\begin{array}{l}379.12(104) \\
p<0.001\end{array}$ & $\begin{array}{l}283.57(101) \\
p<0.001\end{array}$ \\
\hline SRMR & 0.059 & 0.056 & 0.047 & 0.045 & 0.080 & 0.070 \\
\hline RMSEA (90\% Cl) & $\begin{array}{l}0.086 \\
(0.078-0.095)\end{array}$ & $\begin{array}{l}0.078 \\
(0.069-0.087)\end{array}$ & $\begin{array}{l}0.066 \\
(0.057-0.076)\end{array}$ & $\begin{array}{l}0.064 \\
(0.055-0.074)\end{array}$ & $\begin{array}{l}0.118 \\
(0.112-0.124)\end{array}$ & $\begin{array}{l}0.103 \\
(0.097-0.110)\end{array}$ \\
\hline CFI & 0.951 & 0.966 & 0.975 & 0.979 & 0.923 & 0.949 \\
\hline NNFI (TLI) & 0.940 & 0.955 & 0.969 & 0.973 & 0.911 & 0.939 \\
\hline \multicolumn{7}{|l|}{ Log-likelihoods } \\
\hline$-2 \ln (L)(e p)$ & $6857(24)$ & $6787(27)$ & $6718(24)$ & $6696(27)$ & 8105 (32) & 7851 (35) \\
\hline AIC (ep) & $6905(24)$ & $6841(27)$ & $6766(24)$ & $6750(27)$ & 8169 (32) & $7921(35)$ \\
\hline BIC (ep) & 7014 (24) & $6964(27)$ & $6874(24)$ & $6872(27)$ & $8313(32)$ & 8078 (35) \\
\hline \multicolumn{7}{|l|}{ Reliability } \\
\hline Coefficient $\mathrm{H}$ & 0.826 & $0.663 / 0.608 / 0.713$ & 0.822 & $0.591 / 0.599 / 0.622$ & 0.882 & $0.823 / 0.720 / 0.667$ \\
\hline
\end{tabular}

Note. The table shows goodness of fit (GOF) indexes, log-likelihood and reliability using confirmatory factor analysis (CFA) when treating the various short versions as one- and three-dimensional. CFA was performed using LISREL software

SB scaled $x^{2}$ :Satorra-Bentler scaled chi-square. SRMR (standardised root mean square residual) and RMSEA (root-mean-squared error of approximation): values $<0.05$ indicate good model fit, and values $<0.08$ indicate an acceptable model fit. CFI (comparative fit index) and NNFI (non-normed fit index [or TLI = Tucker and Lewis fit index]): values $>0.95$ indicate good model fit (Hu and Bentler, 1999)

$-2 \ln (\mathrm{L})$ : likelihood function, AIC: Akaike's information criterion, BIC: Bayesian information criterion, ep: number of estimated parameters. Lower values indicate a better overall fit

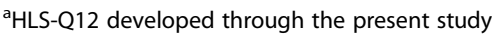

recommends a complex 12-multidimensional approach returning 12 subscale-scores for each individual. This is of little practical use, especially as proficiency cannot be compared across the different subscales owing to relative points of zero. Owing to few items in each subscale, few of the 12 subscales were sufficiently reliable.

Contrary to common practice when using the HLSEU-Q47, we cannot recommend reporting either total or health domain subscale scores. The three-dimensional approach, reflecting the three health domains, obtained better data-model fit than the one-dimensional approaches. The three health domain subscales each returned sufficiently large reliability indexes, but lower indexes than those reported by the HLS-EU Consortium [11], Nakayama et al. [18] and Duong et al. [19].

Prior research on people with chronic disease (type 2 diabetes) [20] indicates that the HC subscale brings notable multidimensionality into the data. It is very interesting that this not was supported in our recent analyses of a national sample, because it indicates that the "health care" subscale actually work differently for patients with chronic disease than in the population as a whole. This seems reasonable, as people with chronic disease have more experiences with using health care facilities than the general population.

\section{Analyses of HLS-EU-Q47 at item-level}

Like previous studies [20, 21] we observed items violating local independence, items displaying DIF, and poorly fitting items. Poorly fitting items which over- or under-discriminate tap into constructs other than the latent trait [64]. Over-discriminating items measure "too much of something else" that is positively correlated with the latent trait and are therefore viewed as less problematic than under-discriminating items. Contrary to Finbråten et al. [20], who found that few items displayed DIF in people with chronic disease (type 2 diabetes), we found several items displaying DIF for age and education, which means that people with higher versus lower levels of education, as well as younger versus older people, probably perceive these items differently. Consequently, comparisons of HL across age groups or educational levels would be invalid [54]. The increased number of items displaying DIF in our study might be expected as the sample of people with type 2 diabetes in Finbråten et al. [20] mainly consisted of elderly persons.

\section{Psychometric properties of the of HLS-Q12, HL-SF12 and HLS-EU-Q16 \\ Overall analyses of the of the HLS-Q12, HL-SF12 and HLS- EU-Q16}

Based on the PCA and $t$-test procedures in RUMM, none of the short versions stood out as sufficiently unidimensional. However, considering the lower binominal 95\% CI proportions of 0.07 , the two 12 item versions HLS-Q12 and HL-SF12 could be considered sufficiently unidimensional [54]. Hagell [41] claims that the width of 
Finbråten et al. BMC Health Services Research (2018) 18:506

Page 12 of 17

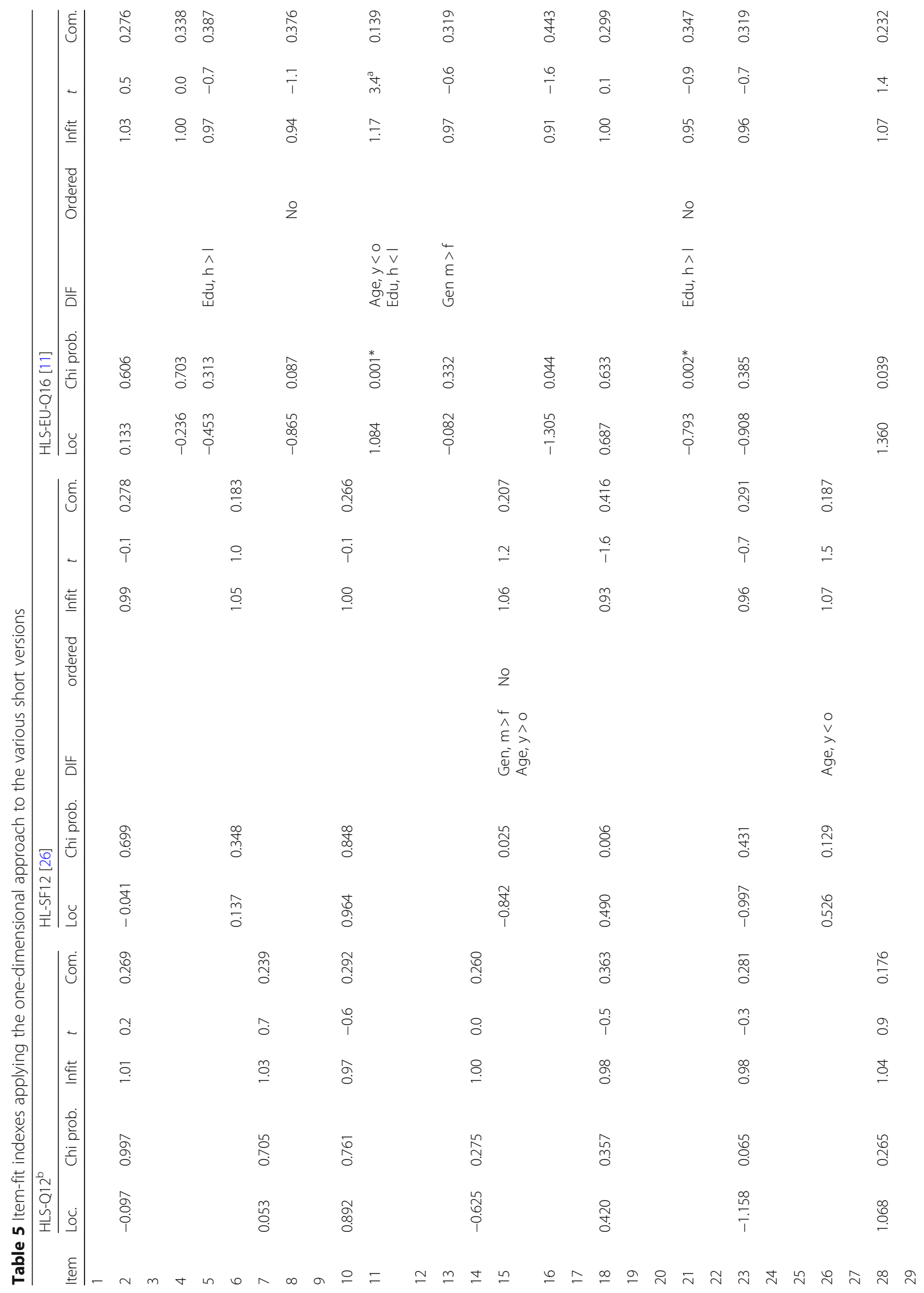




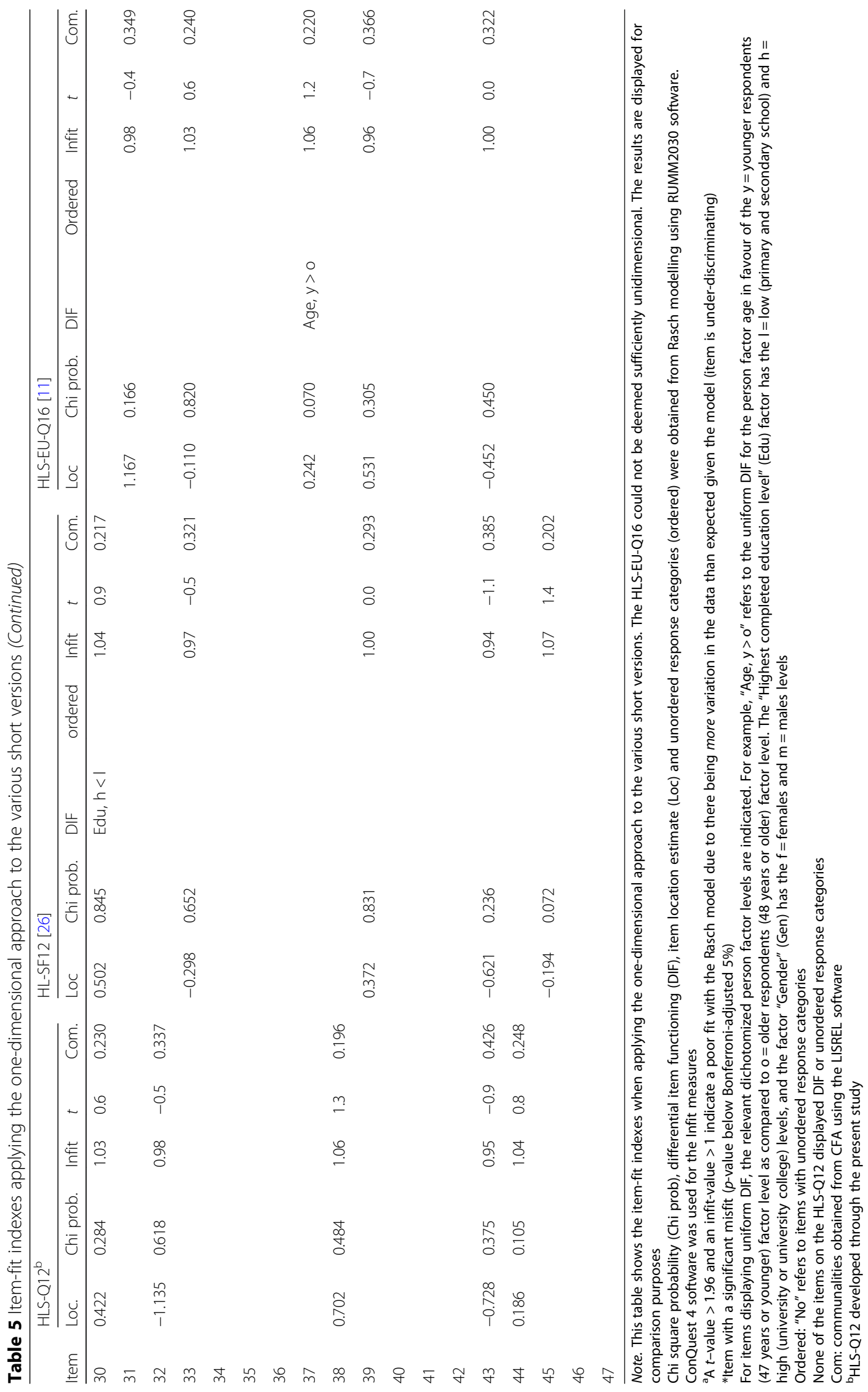


the binominal 95\% CI is influenced by sample size. When investigating dimensionality in a larger sample, binominal 95\% CI-proportions might represent acceptable values. Moreover, unidimensionality is a relative matter and depends on the level of precision. Evaluation of unidimensionality should also include an assessment of the purpose of the measurement, together with theoretical and practical considerations [30, 41].

Comparing the one- and three-dimensional approaches to the short versions, the three-dimensional approach obtained lowest deviance and sufficient values for the GOF indexes. Like some previous studies [32, 65] we found that Rasch modelling and CFA returned similar results. Thus, a three-dimensional approach could be recommended for the short versions. However, when applying a three-dimensional approach the three subscales obtained low reliability indexes. The difference in deviance between the one- and three-dimensional approaches was significant; but the differences were rather small for the two 12 item versions.

A one-dimensional approach to the HLS-Q12 and HL-SF12 could probably be defensible as the results point to minor violations of unidimensionality. Further, the one-dimensional approach yields higher reliability indexes than each of the subscales of the three-dimensional approach. Providing one HL score for each individual, the one-dimensional approach to the HLS-Q12 and the HL-SF12 is practical from a clinical point of view. In addition, the HLS-Q12 and HL-SF12 are in line with the conceptual model of Sørensen et al. [16], but dimensionality should be further explored in future studies.

\section{Comparing various short versions}

Comparing the psychometric properties of the various short versions, the HLS-Q12 obtained best fit to the Rasch model, whereas the HL-SF12 obtained best fit through CFA. The HL-SF12 was found to have several weaknesses, including items with unordered response categories (item 15) and several items displaying DIF (items 15, 26 and 30). Owing to a larger number of items, the HLS-EU-Q16 stood out as more reliable than the other short versions [63]. However, the HLS-EU-Q16 could not be considered sufficiently unidimensional, it had the highest AIC, and did not yield acceptable GOF indexes. In addition, problems regarding item misfit, DIF and unordered response categories were observed.

Altogether, the HLS-Q12 has better psychometric properties than the HL-SF12 and the HLS-EU-Q16. The HLS-Q12 can be deemed the best-targeted scale and free from under-discriminating items and DIF. The HLS-Q12 reflects the conceptual framework, and it is well balanced because it consists of one item from each of the 12 dimensions. The HLS-Q12 version could be well suited for use in HL screenings at both the individual and community levels. Nevertheless, in future studies, we recommend extending the number of response categories from four to six to increase the reliability of the scale [66]. However, item 38 represents a specific concern because this item under-discriminated when applying one-, three- and 12-dimensional approaches to the entire HLS-EU-Q47. In contrast, when we applied a one-dimensional approach to the HLS-Q12, the item showed good model fit. In future validation of the HLS-Q12, this item should be further investigated.

\section{Item difficulty}

Using the HLS-Q12, items 28 (judge if the information on health risks in the media is reliable) and 10 (judge the advantages and disadvantages of different treatment options) were the hardest items to endorse. Consequently, people may use information that is not evidence-based and potentially harmful to their health. Hence, health professionals should guide individuals in accessing valid and reliable health information. Further, health professionals should help individuals to develop the ability to critically assess health information from different sources. People also need guidance in judging the advantages and disadvantages of various treatment options. Low HL might cause difficulties with promoting and taking responsibility for one's own health, as well as participating in shared descision-making regarding health issues. According to Nutbeam [67], nurses and other health professionals must become aware of the effects of low HL. Hence, nurses and other health professionals should map HL in their patients and adapt health communications to the individual or target group.

Further research on HL among Norwegians is needed, especially research examining the correlation between $\mathrm{HL}$ and health behaviour and the correlation between access to and the use of health services. It would also be interesting to compare HL across cultures and nationalities. However, before comparing HL across nations and cultures, DIF analyses should be performed to investigate the effects of cultural differences on participants' interpretations of the content of the items.

\section{Limitations}

Ipsos, the agency that performed the sampling and data collection, guarantees representativeness. However, the educational level of this sample was higher than that of the average Norwegian population. In this study, more than half of participants had education at the university level, while this is true of only $33 \%$ of the general population [68].

Although around 1000 individuals were recruited from each of the included EU countries, 900 individuals were included in this study. In Rasch modelling, there is no exact recommendations for sample size. However, Linacre 
[69] recommends 250 individuals for polytomous data and 10 extra individuals per response category. Hair [70] recommends at least 300 individuals when performing CFA. According to these recommendations, a sample size of 900 would be sufficient.

The HLS-Q12 was developed based on the results of Rasch modelling of the HLS-EU-Q47 using two populations, people with type 2 diabetes [20] and the general Norwegian population. However, its further application to other populations will yield more evidence regarding the validity and reliability of the scale. Future validation should be performed using multinational data.

\section{Conclusions}

The HLS-EU-Q47 was found to best fit a 12-dimensional model, which indicates that a multidimensional approach should be applied when the entire HLS-EU-Q47 is used to measure HL. Consequently, it is not statistically defensible to calculate total HL scores for individuals on the basis of the HLS-EU-Q47, and the estimate of HL for a person cannot be derived from his or her raw score on the HLS-EU-Q47. Relying on 12 different but related scale scores for individuals may be impractical from a clinical point of view. Several items on the HLS-EU-Q47 showed misfit, DIF or unordered response categories, which indicates the need to revise the scale. One should be careful in implementing HL actions based on scores obtained via the HLS-EU-Q47. Hence, instruments should be thoroughly validated before being used in large-population surveys.

This study showed that the HLS-EU-Q47 suffers under particular weaknesses. However, our parsimonious HLS-Q12 meets the assumptions and the requirements of objective measurement while still reflecting the conceptual HL model scaffolding the HLS-EU-Q47. Health professionals aiming to adapt their communication to patients' HL will obviously benefit from a measurement scale like the HLS-Q12 as it could be considered a clinically feasible screening instrument, which does not require advanced on-site psychometric methods. The economic gains that might lie in rationalising health care by applying the short but sufficient HLS-Q12 is relevant for the development of health policies. Researchers aiming to understand which factors impact $\mathrm{HL}$ and how $\mathrm{HL}$ is related to health outcomes will definitely benefit from an HL scale with sufficient psychometric quality. More importantly, the conclusions from such research have the potential to feed further information back into policymaking.

\section{Abbreviations}

A: accessing health information; AIC: Akaike's information criterion; ANOVA: analyses of variance; B: understanding health information; BIC: Bayesian information criterion; $\mathrm{C}$ : appraising health information CFA: confirmatory factor analysis; CFI: comparative fit index; Cl: confidence interval; D: applying health information; DIF: differential item functioning; DP: disease prevention; ep: estimated parameters; GOF: goodness of fit; HC: healthcare; HL: health literacy; HLS-EU-Q47: European Health Literacy Survey Questionnaire; HP: health promotion; LRT: likelihood ratio test statistics; ML: maximum likelihood estimation; MNSQ: mean square; NNFI: non-normed fit index; PCA: principal component analysis; PRM: polytomous Rasch model; PSI: person separation index; PSR: person separation reliability; RMSEA: root-mean-squared error of approximation; SB scaled $x^{2}$ : Satorra-Bentler scaled chi-square; SRMR: standardised root mean square residual; TLI: Tucker and Lewis fit index

\section{Acknowledgements}

The authors thank Kristine Sørensen and the HLS-EU Consortium for their permission to use the HLS-EU-Q47 in this study.

\section{Funding}

The data collection was funded by the Norwegian Nurses' Organisation, Inland Norway University of Applied Sciences and the Public Health Nutrition research group at Oslo Metropolitan University.

\section{Availability of data and materials}

The data set used and analysed in the current study is available from the corresponding author upon reasonable request.

\section{Authors' contributions}

All authors (HSF, BWL, GN, KSP, AT and ØG) contributed to the study design. HSF, KSP and $\varnothing \mathrm{G}$ translated the instrument. HSF and KSP initiated the data collection. HSF and ØG performed the Rasch modelling and CFA. HSF wrote the draft of the manuscript. All authors (HSF, BWL, GN, KSP, AT and ØG) contributed to reading, revising and approving the final manuscript.

\section{Ethics approval and consent to participate}

This study was approved by the Norwegian Social Science Data Service (NSD), ref. 38,917. Subject participation was voluntary, and questionnaires were completed anonymously. As data were collected using telephone interviews, verbal informed consent was obtained from the participants. This procedure was approved by the NSD.

\section{Competing interests}

The authors declare that they have no competing interests.

\section{Publisher's Note}

Springer Nature remains neutral with regard to jurisdictional claims in published maps and institutional affiliations.

\section{Author details}

${ }^{1}$ Department of Public Health and Department of Nursing, Faculty of Social and Health Sciences, Inland Norway University of Applied Sciences, PO Box 400, N-2418 Elverum, Norway. ${ }^{2}$ Department of Health Sciences, Faculty of Health, Science and Technology, Nursing science, Karlstad University, SE-65188 Karlstad, Sweden. ${ }^{3}$ Department of Nursing, Faculty of Social and Health Sciences, Inland Norway University of Applied Sciences, PO Box 400, $\mathrm{N}-2418$ Elverum, Norway. ${ }^{4}$ Department of Nursing and Health Promotion, Faculty of Health Sciences, OsloMet - Oslo Metropolitan University, PO Box 4, St Olavs plass, N-0130 Oslo, Norway. ${ }^{5}$ Norwegian Centre for Science Education, Faculty of Mathematics and Natural Sciences, University of Oslo, PO Box 1106, Blindern, N-0317 Oslo, Norway.

Received: 2 November 2017 Accepted: 4 June 2018 Published online: 28 June 2018

\section{References}

1. Kickbusch I, Pelikan JM, Apfel F, Tsouros AD (eds.). Health Literacy. The solid facts. Copenhagen: WHO Regional Office for Europe; 2013. http://www. euro.who.int/_data/assets/pdf_file/0008/190655/e96854.pdf . Accessed 8 June 2017.

2. Vandenbosch J, Van den Broucke S, Vancorenland S, Avalosse H, Verniest R, Callens M. Health literacy and the use of healthcare services in Belgium. J Epidemiol Community Health. 2016;70(10):1032-8.

3. Sørensen K, Pelikan JM, Rothlin F, Ganahl K, Slonska Z, Doyle G, Fullam J, Kondilis B, Agrafiotis D, Uiters E, et al. Health literacy in Europe: comparative 
results of the European health literacy survey (HLS-EU). Eur J Pub Health. 2015;25(6):1053-8

4. Berkman ND, Sheridan SL, Donahue KE, Halpern DJ, Crotty K. Low health literacy and health outcomes: an updated systematic review. Ann Intern Med. 2011;155(2):97.

5. Bostock S, Steptoe A. Association between low functional health literacy and mortality in older adults: longitudinal cohort study. BMJ 2012; 314:e1602.

6. von Wagner C, Knight K, Steptoe A, Wardle J. Functional health literacy and health-promoting behaviour in a national sample of British adults. J Epidemiol Community Health. 2007;61(12):1086-90.

7. Fernandez DM, Larson JL, Zikmund-Fisher BJ. Associations between health literacy and preventive health behaviors among older adults: findings from the health and retirement study (report). BMC Public Health. 2016;16(1):596.

8. Jayasinghe UW, Harris MF, Parker SM, Litt J, van Driel M, Mazza D, Del Mar C, Lloyd J, Smith J, Zwar N. The impact of health literacy and life style risk factors on health-related quality of life of Australian patients. Health Qual Life Outcomes. 2016;14(1):68.

9. Palumbo R, Annarumma C, Adinolfi P, Musella M, Piscopo G. The Italian health literacy project: insights from the assessment of health literacy skills in Italy. Health Policy. 2016;120(9):1087-94.

10. Geboers B, Reijneveld SA, Jansen CJM, de Winter AF. Health literacy is associated with health behaviors and social factors among older adults: results from the LifeLines cohort study. J Health Commun. 2016;21(Suppl 2):45-53.

11. HLS-EU Consortium. Comparative report of health literacy in eight EU member states. The European Health Literacy Survey HLS-EU. 2012;

12. Levin-Zamir D, Baron-Epel OB, Cohen V, Elhayany A. The association of health literacy with health behavior, socioeconomic indicators, and selfassessed health from a national adult survey in Israel. J Health Commun. 2016;21(Suppl 2):61-8.

13. Parker RM, Baker DW, Williams MV, Nurss JR. The test of functional health literacy in adults: a new instrument for measuring patients' literacy skills. J Gen Intern Med. 1995;10(10):537-41.

14. Davis TC, Long SW, Jackson RH, Mayeaux E, George RB, Murphy PW, Crouch MA. Rapid estimate of adult literacy in medicine: a shortened screening instrument. Fam Med. 1993;25(6):391-5.

15. Sørensen K, Van den Broucke S, Pelikan JM, Fullam J, Doyle G, Slonska Z, Kondilis B, Stoffels V, Osborne RH, Brand H. Measuring health literacy in populations: illuminating the design and development process of the European health literacy survey questionnaire (HLS-EU-Q). BMC Public Health. 2013;13(1):948.

16. Sørensen K, Van den Broucke S, Fullam J, Doyle G, Pelikan J, Slonska Z, Brand $\mathrm{H}$. Health literacy and public health: a systematic review and integration of definitions and models. BMC Public Health. 2012;12(1):1-13.

17. Duong TV, Lin I-F, Sørensen K, Pelikan JM, Van Den Broucke S, Lin Y-C, Chang PW. Health literacy in Taiwan: a population-based study. Asia Pac J Public Health. 2015;27(8):871-80.

18. Nakayama K, Osaka W, Togari T, Ishikawa H, Yonekura Y, Sekido A, Matsumoto M. Comprehensive health literacy in Japan is lower than in Europe: a validated Japanese-language assessment of health literacy. BMC Public Health. 2015;15(1):505

19. Duong TV, Aringazina A, Baisunova G, Nurjanah PTV, Pham KM, Truong TQ Nguyen KT, Oo WM, Mohamad E, et al. Measuring health literacy in Asia: validation of the HLS-EU-Q47 survey tool in six Asian countries. J Epidemiol. 2017;27(2):80-6.

20. Finbråten $\mathrm{HS}$, Pettersen $\mathrm{KS}$, Wilde-Larsson $\mathrm{B}$, Nordström G, Trollvik $\mathrm{A}$, Guttersrud $\varnothing$. Validating the European health literacy survey questionnaire in people with type 2 diabetes. Latent trait analyses applying multidimensional Rasch modelling and confirmatory factor analysis. J Adv Nurs. 2017;73(11):2730-44.

21. Huang Y-J, Chen C-T, Lin G-H, Wu T-Y, Chen S-S, Lin L-F, Hou W-H, Hsieh CL. Evaluating the European health literacy survey questionnaire in patients with stroke: a latent trait analysis using Rasch modeling. Patient. 2018;11(1): 83-93.

22. Toci E, Burazeri G, Sorensen K, Kamberi H, Brand H. Concurrent validation of two key health literacy instruments in a south eastern European population. Eur J Pub Health. 2015;25(3):482-6.

23. Berens $E-M$, Vogt D, Messer M, Hurrelmann K, Schaeffer D. Health literacy among different age groups in Germany: results of a cross-sectional survey. BMC Public Health. 2016;16(1):1151.

24. Schaeffer D, Berens EM, Vogt D. Health literacy in the German population. Deutsches Arzteblatt International. 2017;114(4):53-60.
25. Pleasant A, McKinney J, Rikard R. Health literacy measurement: a proposed research agenda. J Health Commun. 2011;16(Suppl 3):11-21.

26. Duong TV, Chang PW, Yang S-H, Chen M-C, Chao W-T, Chen T, Chiao P. Huang H-L. a new comprehensive short-form health literacy survey tool for patients in general. Asian Nurs Res (Korean Soc Nurs Sci). 2017;11 (1):30-5.

27. Andrich $\mathrm{D}$. Distinctions between assumptions and requirements in measurement in the social sciences. In: Keats JA, Taft R, Heath RA, Lovibond $\mathrm{SH}$, editors. Mathematical and theoretical systems. North Holland: Elsevier science publishers BV; 1989. p. 7-16.

28. Stenner AJ. Specific objectivity - local and general. Rasch Measurement Transactions. 1994;8(3):374

29. Andersen EB. Sufficient statistics and latent trait models. Psychometrika. 1977:42(1):69-81.

30. Andrich D. Rasch models for measurement. Newsbury Park, CA: Sage; 1988.

31. Osteen P. An introduction to using multidimensional item response theory to assess latent factor structures. J Soc Social Work Res. 2010;1(2):66-82.

32. DeMars CE. A comparison of confirmatory factor analysis and multidimensional Rasch models to investigate the dimensionality of testtaking motivation. J Appl Meas. 2013;14(2):179-96.

33. European Countries by Population http://www.worldometers.info/ population/countries-in-europe-by-population/. Accessed 2 May 2018.

34. Masters GN. A Rasch model for partial credit scoring. Psychometrika. 1982; 47(2):149-74.

35. Rasch G. Probabilistic models for some intelligence and attainment tests, expanded edn. Chicago: University of Chicago Press; 1980.

36. Adams RJ, Wilson MR, Wang W-C. The multidimensional random coefficients multinomial logit model. Appl Psychol Meas. 1997;21(1):1-23.

37. Andrich D, Sheridan B, Luo G. Rasch unidimensional measurment model. RUMM2030. In: RUMM laboratory Pty Itd.; 2003.

38. Adams RJ, Wu ML, ACERConQuest WMR. Generalised item response modelling software. 4th ed. Camberwell, Victoria: Australian council for Educational Research; 2015.

39. Jöreskog K, Sörbom D. LISREL 9.3 software. Skokie: Scientific Software International, Inc; 2017.

40. RUMM Laboratory Pty Ltd. Displaying the RUMM 2030 Analysis. Duncraig, Australia: RUMM Laboratory Pty Ltd.; 2012.

41. Hagell P. Testing rating scale unidimensionality using the principal component analysis (PCA)/t-test protocol with the Rasch model: the primacy of theory over statistics. Open J Stat. 2014;4:456-65.

42. Smith EV. Understanding Rasch measurement: detecting and evaluating the impact of multidimenstionality using item fit statistics and principal component analysis of residuals. J Appl Meas. 2002;3:205-31.

43. Horton M, Marais I, Christensen KB. Dimensionality. In: Christensen KB, Kreiner S, Mesbah M, editors. Rasch models in health. Hoboken, NJ USA: John Wiley \& Sons, Inc; 2013.

44. RUMM Laboratory Pty Ltd. Interpreting RUMM2030. Part IV Multidimensionality and subtests in RUMM. Duncraig, Australia: RUMM Laboratory Pty Ltd.; 2009.

45. Adams R, Wu M. Multidimensional models. In ConQuest Tutorial 2010 https:/www.acer.edu.au/files/Conquest-Tutorial-7-MultidimensionalModels. pdf. Accessed 31 Oct 2017.

46. Andrich D, Humphry SM, Marais I. Quantifying local, response dependence between two polytomous items using the Rasch model. Appl Psychol Meas. 2012;36(4):309-24

47. Wilks SS. The large-sample distribution of the likelihood ratio for testing composite hypotheses. Ann Stat. 1938;9(1):60-2.

48. Akaike H. A new look at the statistical model identification. IEEE Trans Autom Control. 1974;19(6):716-23.

49. Allen DD, Wilson M. Introducing multidimensional item response modeling in health behavior and health education research. Health Educ Res. 2006; 21(Suppl 1):i73-84

50. Smith RM. Using item mean squares to evaluate fit to the Rasch model. In: the Annual Meeting of the American Educational Research Association. San Francisco, CA; 1995.

51. Tennant A, Conaghan P. The Rasch measurement model in rheumatology: what is it and why use it? When should it be applied, and what should one look for in a Rasch paper? Arthritis Care Res. 2007;57(8):1358-62.

52. Andrich D. Rating scales and Rasch measurement. Expert Rev Pharmacoecon Outcomes Res. 2011;11(5):571-85.

53. Hagquist C. Evaluating composite health measures using Rasch modelling: an illustrative example. Soz Praventivmed/Soc Prev Med. 2001;46(6):369-78. 
54. Hagquist C, Bruce M, Gustavsson JP. Using the Rasch model in nursing research: an introduction and illustrative example. Int J Nurs Stud. 2009; 46(3):380-93.

55. Bandalos DL, Finney SJ. Factor analysis; exploratory and confirmatory. In: Hancock GR, Mueller RO, Stapleton LM, editors. The reviewer's guide to quantitative methods in the social sciences. New York: Routledge; 2010.

56. Joreskog KG, Olsson UH, Wallentin FY. Multivariate analysis with LISREL. In: Springer international publishing; 2016.

57. Satorra A, Bentler PM. A scaled difference chi-square test statistic for moment structure analysis. Psychometrika. 2001;66(4):507-14.

58. Satorra A, Bentler PM. Ensuring positiveness of the scaled difference chisquare test statistic. Psychometrika. 2010;75(2):243-8.

59. Schumacker RE. Lomax RG. A beginner's guide to structural equation modeling. 3rd ed. New York: Routledge; 2010.

60. Hu Lt BPM. Cutoff criteria for fit indexes in covariance structure analysis: conventional criteria versus new alternatives. Struct Equ Modeling. 1999;6(1):1-55.

61. Brown TA. Confirmatory factor analysis for applied research. New York: Guilford Publications; 2015

62. Hancock GR, Mueller RO. Rethinking construct reliability within latent variable systems. In: Cudeck R, du Toit S, Sorbom D, Lincolnwood IL, editors. Structural equation modeling: present and future - a Festschrift in honor of Karl Joreskog. Scientific software international; 2001. p. 195-216.

63. Frisbie DA. Reliability of scores from teacher-made tests. Educ Meas: Issues Pract. 1988;7(1):25-35.

64. Masters GN. Item discrimination: when more is worse. J Educ Meas. 1988: 25(1):15-29.

65. Wolfe EW, Singh K. A comparison of structural equation and multidimensional Rasch modeling approaches to confirmatory factor analysis. J Appl Meas. 2011;12(3):212-21

66. Preston CC, Colman AM. Optimal number of response categories in rating scales: reliability, validity, discriminating power, and respondent preferences. Acta Psychol. 2000;104(1):1-15.

67. Nutbeam D. Discussion paper on promoting, measuring and implementing health literacy - implications for policy and practice in non-communicable disease prevention and control. World Health Organ. 2017:1-29.

68. Statistics Norway. Befolkningens utdanningsnivå [Educational attainment of the population]. https://www.ssb.no/utdanning/statistikker/utniv/aar. Accessed 2 Oct 2017.

69. Linacre JM. Constructing measurement with a many-facet Rasch model. In: Wilson M, editor. Objective measurement: theory Practice (Vol.2). Newark, NJ: Ablex; 1994

70. Hair JF. Multivariate data analysis. 7th ed. Harlow: Pearson; 2014

Ready to submit your research? Choose BMC and benefit from:

- fast, convenient online submission

- thorough peer review by experienced researchers in your field

- rapid publication on acceptance

- support for research data, including large and complex data types

- gold Open Access which fosters wider collaboration and increased citations

- maximum visibility for your research: over $100 \mathrm{M}$ website views per year

At BMC, research is always in progress.

Learn more biomedcentral.com/submissions 